Homology, Homotopy and Applications, vol.10(1), 2008, pp.327-343

\title{
A NEW HIGHER HOMOTOPY GROUPOID: THE FUNDAMENTAL GLOBULAR $\omega$-GROUPOID OF A FILTERED SPACE
}

\author{
RONALD BROWN
}

(communicated by G. Janelidze)

\begin{abstract}
We show that the graded set of filter homotopy classes rel vertices of maps from the $n$-globe to a filtered space may be given the structure of (strict) globular $\omega$-groupoid. The proofs use an analogous fundamental cubical $\omega$-groupoid due to the author and Philip Higgins in 1981. This method also relates the construction to the fundamental crossed complex of a filtered space, and this relation allows the proof that the crossed complex associated to the free globular $\omega$-groupoid on one element of dimension $n$ is the fundamental crossed complex of the $n$-globe.
\end{abstract}

\section{Contents}

1 Introduction $\quad 328$

2 Disks, globes, and cubes 332

3 The free globular $\omega$-groupoid on one generator 334

4 The higher homotopy van Kampen theorem 335

5 Monoidal closed structures $\quad 336$

6 Nerve and classifying space of globular $\omega$-groupoids 337

\begin{tabular}{|ll|}
\hline A The globular site & 338
\end{tabular}

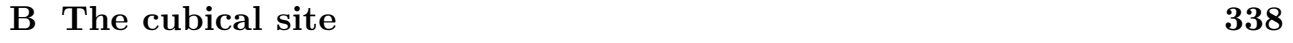

$\begin{array}{ll}\text { References } & 341\end{array}$

The author was supported for part of this work by a Leverhulme Emeritus Fellowship (2002-2004). Received February 23, 2007, revised February 11, 2008; published on April 18, 2008.

2000 Mathematics Subject Classification: 18D10,18G30,18G50,20L05,55N10,55N25.

Key words and phrases: filtered space, higher homotopy groupoid, higher homotopy van Kampen theorem, cubical singular complex, free globular groupoid.

This article is available at http://intlpress.com/HHA/v10/n1/a14

Copyright (C) 2008, Ronald Brown. Permission to copy for private use granted. 


\section{Introduction}

By the $n$-globe $G^{n}$ we mean the subspace of Euclidean $n$-space $\mathbb{R}^{n}$ of points $x$ such that $\|x\| \leqslant 1$ but with the cell structure for $n \geqslant 1$

$$
G^{n}=e_{ \pm}^{0} \cup e_{ \pm}^{1} \cup \cdots \cup e_{ \pm}^{n-1} \cup e^{n} .
$$

This structure will be given precisely in Section 2 .
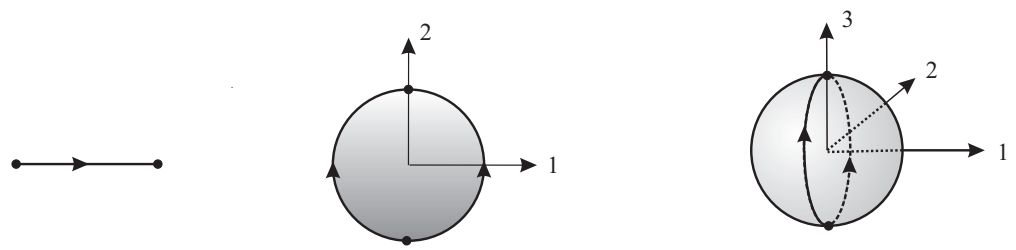

A filtered space is a compactly generated space $X_{\infty}$ and a sequence of subspaces

$$
X_{*}: X_{0} \subseteq X_{1} \subseteq \cdots \subseteq X_{n} \subseteq \cdots \subseteq X_{\infty} .
$$

A map of filtered spaces $f: Y_{*} \rightarrow X_{*}$ is a map $f: Y_{\infty} \rightarrow X_{\infty}$ such that $f\left(Y_{n}\right) \subseteq X_{n}$ for all $n \geqslant 0$. This gives the category FTop of filtered spaces. A filter homotopy $f_{t}: f_{0} \simeq f_{1}$ is a continuous family of filtered maps $f_{t}: Y_{*} \rightarrow X_{*}$ for $0 \leqslant t \leqslant 1$. This is to be contrasted with a homotopy of filtered maps which has the requirement $f_{t}\left(Y_{n}\right) \subseteq X_{n+1}$ for all $t$ and $n \geqslant 0$.

The $n$-globe $G^{n}$ has a skeletal filtration giving a filtered space $G_{*}^{n}$. If $X_{*}$ is a filtered space then we have a globular singular complex $R^{\circ} X_{*}$, which in dimension $n$ is $\mathrm{FTop}\left(G_{*}^{n}, X_{*}\right)$. In Appendix $\mathrm{A}$ we will explain the structure of $R^{\circ} X_{*}$ as a globular set.

We define

$$
\rho^{\circ} X_{*}=\left(R^{\circ} X_{*} / \equiv\right),
$$

where $\equiv$ is the relation of filter homotopy rel vertices. It is clear that $\rho^{\circ} X_{*}$ inherits from $R^{\circ} X_{*}$ the structure of globular set. Our main result is the following:

Theorem 1.1 (Main Theorem). For $n \geqslant 1$ there are compositions $\circ_{i}, 1 \leqslant i \leqslant n$ in dimension $n$ giving the globular set $\rho^{\circ} X_{*}$ the structure of strict globular $\omega$-groupoid.

We call $\rho^{\circ} X_{*}$ the fundamental globular higher homotopy groupoid of the filtered space $X_{*}$. The proof of this theorem goes via the notion of cubical higher homotopy groupoid of a filtered space, established in $[\mathbf{B H 8 1 b}$. It should be useful therefore to put these results in context.

A general characterisation of work on higher homotopy groupoids in which the author has been involved may be subsumed in the following diagram of categories and functors and its properties: 


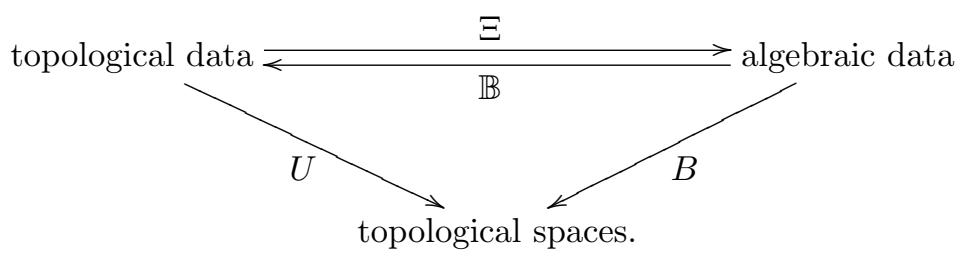

It has been possible to give categories of 'topological data', of 'algebraic data' and functors as above, where $U$ is the forgetful functor and $B=U \circ \mathbb{B}$, with the following properties:

(1) the functor $\Xi$ is defined homotopically and satisfies a higher homotopy van Kampen theorem $(\mathrm{HHvKT})^{1}$, in that it preserves certain colimits;

(2) $\Xi \circ \mathbb{B}$ is naturally equivalent to 1 ;

(3) there is a natural transformation $1 \rightarrow \mathbb{B} \circ \Xi$ preserving some homotopical information.

The purpose of (1) is to allow some calculation of $\Xi$ by gluing simple examples, such as convex subsets, following the use of the fundamental groupoid in Bro06. This condition (1) at present also rules out some widely used algebraic data, such as for example simplicial groups or groupoids, or differential graded algebras, since for those cases no such functor $\Xi$ is known. (2) shows that the algebraic data faithfully captures some of the topological data. The imprecise (3) gives further information on the algebraic modelling. The functor $B$ should be called a classifying space because it often generalises the classifying space of a group or groupoid. It has also been found useful in the homotopy classification of maps.

Here is a table of examples that have been found.

\begin{tabular}{|c|c|}
\hline Topological data & Algebraic data \\
\hline \hline space with base point & groups \\
\hline space with set of base points & groupoids \\
\hline pointed pair of spaces & crossed modules $^{\text {crossed complexes }}$ \\
\hline filtered space & cat $^{n}$-groups \\
\hline$n$-cube of pointed spaces & crossed $n$-cube of groups \\
\hline$n$-cube of pointed spaces
\end{tabular}

Strong results in the last two cases are shown in BL87, ES87.

In this paper we will deal only with the case of filtered spaces, which of course includes the first three cases. There are still further choices of algebraic data as shown in the following diagram of equivalent categories, which is taken from [Bro99]:

\footnotetext{
${ }^{1} \mathrm{Jim}$ Stasheff has suggested this term to the author, instead of the previously used Generalised van Kampen Theorem, to make clear the higher homotopy information contained in theorems of this type.
} 


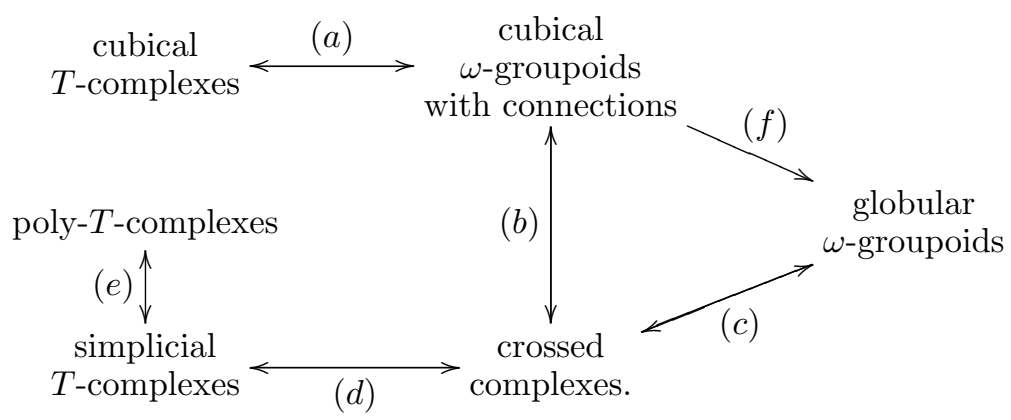

Each arrow here denotes an explicit functor which is an equivalence of categories. The equivalences $(a)$ and $(b)$ are in BH81a; $; a)$ is an essential technical tool in the use of cubical $\omega$-Gpds. The equivalence $(c)$ is in $[$ BH81c], and this with $(b)$ implies the equivalence $(f)$. A direct form of this equivalence is given in the much harder category case in AABS02. The equivalence $(d)$ is due to Ashley in Ash88. The equivalence $(e)$ is due to Jones Jon88. The different forms of algebra reflect different geometries, those of disks, globes, simplices, cubes, as shown in dimension 2 in the following diagram:
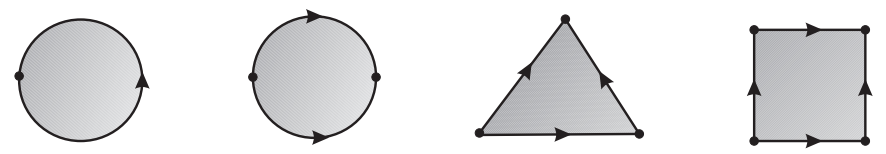

It is because the geometry of convex sets is so much more complicated in dimensions $>1$ than in dimension 1 that new complications emerge for the theories of higher order group theory and of higher homotopy groupoids.

A classical homotopical functor on filtered spaces is the fundamental crossed complex $\Pi X_{*}$ of a filtered space, defined using relative homotopy groups (in the case $X_{0}$ is a singleton) by Blakers [Bla48. Major achievements of the papers [BH81a, BH81b] were

- to define a homotopical functor, which here we call $\rho^{\square}$, from filtered spaces to cubical $\omega$-groupoids with connections (and hence also to cubical $T$-complexes), which in dimension $n$ is the filter homotopy classes rel vertices of filtered maps $I_{*}^{n} \rightarrow X_{*}$ (but see Remark 2.3 );

- to prove that this functor preserved certain colimits;

- to relate $\rho^{\square}$ with the classical functor $\Pi$ from filtered spaces to crossed complexes, and so to prove that $\Pi$ preserves certain colimits.

The proofs do not involve traditional techniques such as singular homology or simplicial approximation. The results give nonabelian information on second relative homotopy groups using crossed module theory (see a survey in [Bro99]), and in higher dimensions give information on the action of the fundamental group. In particular 
the HHvKT has as a corollary a previously unnoticed homotopical excision theorem which has the Relative Hurewicz Theorem as a corollary [BH81b, Example 6, p. 34], while extra information on monoidal closed structures gives the Homotopy Addition Lemma for a simplex [BS07], and homotopy classification theorems [BH91.

Analogous methods to those of $\mathbf{B H 8 1 b}$ were used by Ashley in $\mathbf{A s h 8 8}$ to define a functor $\rho^{\Delta}$ from filtered spaces to simplicial $T$-complexes. His ideas contributed to $[\mathbf{B H 8 1 b}$, and his results are applied in [FMa07.

However, there has been a lack of a directly defined homotopical functor from filtered spaces to globular $\omega$-groupoids, and this gap will be filled in this paper.

The definition of classifying space is convenient via well-developed simplicial constructions. In this way we get the classifying space of a crossed complex BH91. Its properties are further exploited in, for example, BGPT, FMa07, FMP06. However the exposition in BHS08 adopts an earlier cubical approach.

The equivalence of the category of globular $\omega$-groupoids with the category of cubical $\omega$-groupoids with connection, and the monoidal closed structure on the latter constructed in [BH87, imply a monoidal closed structure on the category of globular $\omega$-groupoids. Further, it is shown in BH91] that the simple rule $[f] \otimes[g] \mapsto[f \otimes g]$ gives a natural transformation

$$
\rho^{\square} X_{*} \otimes \rho^{\square} Y_{*} \rightarrow \rho^{\square}\left(X_{*} \otimes Y_{*}\right)
$$

for any filtered spaces $X_{*}, Y_{*}$, where $X_{*} \otimes Y_{*}$ is the usual tensor product of filtered spaces given by

$$
\left(X_{*} \otimes Y_{*}\right)_{n}=\bigcup_{p+q=n} X_{p} \times Y_{q} .
$$

The induced transformation on crossed complexes is shown in BB93 to be an isomorphism if $X_{*}, Y_{*}$ are connected (Definition 4.1) and cofibred (each inclusion $X_{n} \rightarrow X_{r}$, $r>n \geqslant 0$, is a closed cofibration). It follows from the above that there is a natural transformation

$$
\rho^{\circ} X_{*} \otimes \rho^{\circ} Y_{*} \rightarrow \rho^{\circ}\left(X_{*} \otimes Y_{*}\right) .
$$

This natural transformation, which is difficult to construct directly, may be used to enrich the category of filtered spaces over the monoidal closed category of globular $\omega$-groupoids.

It should be apparent from the above that it is the cubical case which gives the intuition and power in formulating and proving these theorems; the basic reason is that cubical theory is handy for: subdivision and its inverse, multiple compositions, the notion of commutative cube, and is also good for tensor products and higher homotopies. Many theorems can then, by equivalences of categories, be translated to the other cases. However the proofs for the cubical cases, particularly the properties of thin elements and $T$-complexes, involve also the use of crossed complexes and the equivalence of categories $(a),(b)$ of diagram (5). Crossed complexes also have a well-developed homotopy theory $\mathbf{B G 8 9}$, and they have a clear relation with chain complexes with operators [BH90]. 
The relation with simplicial theory is useful because of the wide development of simplicial theory. Finally, the relation with the globular theory could be useful because of the wide familiarity of uses of weak structures and lax functors and natural transformations. For example, compare the discussion of Schreier theory using crossed complexes in BH82, BP96, with the use of 2-groupoids in BBF05. Calculational applications are usually made using crossed complexes. For example, the paper [BP96] uses the notion of small free crossed resolution to give calculations which yield small parametrisations of some nonabelian extensions of groups, whereas the notion of free globular $\omega$-groupoid is lacking, or undeveloped.

\section{Disks, globes, and cubes}

Our results follow from an analysis of the relations between globes and cubes. These results are probably well known but need to be done carefully for our purposes.

We give real space $\mathbb{R}^{n}$ the Euclidean norm $\|x\|^{2}=x_{1}^{2}+x_{2}^{2}+\cdots+x_{n}^{2}$. We embed $\mathbb{R}^{n}$ in $\mathbb{R}^{n+1}$ as usual by $x \mapsto(x, 0)$. The $n$-cube $I^{n}$ will be the subset of $\mathbb{R}^{n}$ of points $x$ such that $\left|x_{i}\right| \leqslant 1$ for all $i$. Thus $I=I^{1}$ is identified with $[-1,1]$ and we also identify $I^{n}$ with the $n$-fold product of $I$ with itself.

The $n$-disk is the subspace $D^{n}$ of $\mathbb{R}^{n}$ of points $x$ with $\|x\| \leqslant 1$. The $(n-1)$-sphere $S^{n-1}$ is the subspace of $D^{n}$ of points $x$ with $\|x\|=1$.

We define the $n$-globe $G^{n}$ to be $D^{n}$ as a space, but with the cell structure

$$
G^{n}=e_{ \pm}^{0} \cup e_{ \pm}^{1} \cup \cdots \cup e_{ \pm}^{n-1} \cup e^{n} .
$$

Here for $i<n$ the closed cell $\bar{e}_{ \pm}^{i}$ is the set of points $x=\left(x_{1}, \ldots, x_{n}\right) \in G^{n}$ such that $\|x\|=1, x_{j}=0$ for $j<n-i$ and $\pm x_{n-i} \geqslant 0$. This convention is in keeping with the relationship with cubes which we find convenient. Note that the $(n-1)$-skeleton of $G^{n}$ is contained in $S^{n-1}$.

For each of $Q=\Delta, \square$, o we have a singular complex $S^{Q} X$ of a topological space $X$, giving the well-known simplicial and cubical singular complex, and also a 'globular' singular complex consisting of maps $G^{n} \rightarrow X$. We will later describe this as a 'globular set'.

Definition 2.1. We now define by induction the maps $\phi_{n}: I^{n} \rightarrow G^{n}, n \geqslant 1$, with the following properties, for $x=\left(x_{1}, \ldots, x_{n}\right) \in I^{n}$ :

(i) $\phi_{1}\left(x_{1}\right)=x_{1}$;

(ii) $\left|x_{i}\right|=1$ for some $i=1, \ldots, n$ if and only if $\left\|\phi_{n}(x)\right\|=1$;

(iii) $\left|x_{i}\right|=1$ for some $i=1, \ldots, n$ implies $\left(\phi_{n}(x)\right)_{j}=0$ for $j<i$.

We set for $x=(t, y) \in I \times I^{n-1}$ :

$$
\phi_{n}(t, y)=\left(t \sqrt{1-\left\|\phi_{n-1}(y)\right\|^{2}}, \phi_{n-1}(y)\right) .
$$

First note that if $x=(t, y)$, then

$$
\left\|\phi_{n}(x)\right\|^{2}=t^{2}+\left(1-t^{2}\right)\left\|\phi_{n-1}(y)\right\|^{2} .
$$


This easily proves (ii) and (iii) by induction.

The maps $\phi_{n}: I^{n} \rightarrow G^{n}$ induce a map $\bar{\phi}: S^{\circ} X \rightarrow S^{\square} X$.

We define the globular subset $\gamma K$ of a cubical set $K$ to agree with $K$ in dimensions 0,1 and to be in dimension $n \geqslant 2$ the set of $k$ such that $\partial_{i}^{ \pm} k \in \operatorname{Im} \varepsilon_{1}^{i-1}, i=2, \ldots, n$.

Proposition 2.2. The image of $\bar{\phi}: S^{\circ} X \rightarrow S^{\square} X$ is exactly the globular subset of $S^{\square} X$.

Proof. We prove by induction from the formula for $\phi_{n}$ that the image is globular. Let $p_{1}^{i}: \mathbb{R}^{n} \rightarrow \mathbb{R}^{n-i}$ be the projection omitting the first $i$ coordinates. Suppose that $\phi_{n-1} \bar{\partial}_{i}^{ \pm}=f_{n-1} p_{1}^{i-1}$. Then $\phi_{n} \bar{\partial}_{i+1}^{ \pm}=f_{n-1}^{\prime} p_{1}^{i}$ where $f_{n-1}^{\prime}(x)=\left(0, f_{n-1}(x)\right)$.

For the converse, we prove by induction that these are the only identifications that $\phi_{n}$ makes. Suppose $\phi_{n}(t, y)=\phi_{n}\left(t^{\prime}, y^{\prime}\right)$. Then $\phi_{n-1}(y)=\phi_{n-1}\left(y^{\prime}\right)$ and

$$
t \sqrt{1-\left\|\phi_{n-1}(y)\right\|^{2}}=t^{\prime} \sqrt{1-\left\|\phi_{n-1}\left(y^{\prime}\right)\right\|^{2}} .
$$

Thus if $\left\|\phi_{n-1}(y)\right\| \neq 1$ then $t=t^{\prime}$. But $\left\|\phi_{n-1}(y)\right\|=1$ implies some $\left|y_{i}\right|=1$, by the inductive hypothesis.

Let $X_{*}$ be a filtered space. Then we obtain three filtered singular complexes $R^{Q} X_{*}$ for $Q=D, \circ, \square$ defined as graded sets by

$$
\left(R^{Q} X_{*}\right)_{n}=\mathrm{FTop}\left(Q_{*}^{n}, X_{*}\right) .
$$

There are also associated graded homotopy sets $\rho^{Q} X_{*}$, which in dimension $n$ are given by the quotient maps

$$
p^{Q}: R^{Q}, X_{*} \rightarrow \rho^{Q} X_{*}=R^{Q} X_{*} / \equiv,
$$

where $\equiv$ is the relation of homotopy rel vertices through filtered maps.

In the cases $Q=D, \square$ it is known that these graded sets obtain additional structure giving us, for $Q=D$, the fundamental crossed complex $\Pi X_{*}$, and, for $Q=\square$, what is called in BH81b the fundamental (cubical) $\omega$-groupoid (with connections) of $X_{*}$. However the proof that the standard compositions on $R^{\square} X_{*}$ are inherited by $\rho^{\square} X_{*}$ is nontrivial, as is the crucial result that $p^{\square}$ is a Kan fibration of cubical sets.

Remark 2.3. In $\mathbf{B H 8 1 b}$, the homotopies are not taken rel vertices and a condition $J_{0}$ is imposed, so that each map $\dot{I}^{2} \rightarrow X_{0}$, where $\dot{I}^{2}$ is the boundary of $I^{2}$, may be extended to a map $I^{2} \rightarrow X_{1}$. This condition is in many ways inconvenient. The filling processes used in the proofs can all be started by assuming instead that the homotopies are rel vertices so that the maps $\dot{I}^{2} \rightarrow X_{0}$ required to be extended are in fact all constant. The details will be available in [BHS08].

Our first main result is:

Theorem 2.4. The induced map

$$
\phi^{*}: \rho^{\circ} X_{*} \rightarrow \rho^{\square} X_{*}
$$

is injective. 
Proof. Let $[\alpha],[\beta] \in\left(\rho^{\circ} X_{*}\right)_{n}$ be such that $\phi^{*}[\alpha]=\phi^{*}[\beta]$; that is

$$
[\alpha \phi]=[\beta \phi]
$$

in $\left(\rho^{\square} X_{*}\right)_{n}$. Let $H: \alpha \phi \equiv \beta \phi$ be such a homotopy. Then $H$ is a map $I_{*}^{n+1} \rightarrow X_{*}$ such that writing $I^{n+1}=I^{n} \times I$, each $H_{t}: I_{*}^{n} \rightarrow X_{*}$ is a filtered map.

We use a folding map $\Phi: I^{n} \rightarrow I^{n}$ given by Definition 3.1 of [AABS02] (see Definition $(B .2)$ which has the property that $\Phi$ factors through $\phi$.

We now define a new homotopy $K_{t}=\Phi H_{t}: I_{*}^{n} \rightarrow X_{*}$. Then $K_{t}$ is a globular homotopy $\Phi \alpha \phi \equiv \Phi \beta \phi$. But, by assumption, $\alpha \phi, \beta \phi$ are already globular maps. So the proof is completed with the following lemma.

Lemma 2.5. If $a: I_{*}^{n} \rightarrow X_{*}$ is a globular map, then $\Phi$ a is globularly equivalent to a.

Proof. Since $\Phi$ is a composition of the folding operations $\psi_{i}$, it is sufficient to prove that $\psi_{i} a \equiv_{\mathrm{O}} a$. We follow the proof of [AABS02, Proposition 3.4]. By the definition of $\psi_{i}$ :

$$
\psi_{i} a=\Gamma_{i}^{+} \partial_{i+1}^{-} a \circ_{i+1} a \circ_{i+1} \Gamma_{i}^{-} \partial_{i+1}^{+} a .
$$

But $\partial_{i+1}^{-} a$ and $\partial_{i+1}^{+} a$ are globular. From the laws in Appendix B, we obtain, since $a$ is globular,

$$
\Gamma_{i}^{\mp} \partial_{i+1}^{ \pm} a \in \operatorname{Im} \Gamma_{i}^{\mp} \varepsilon_{i}=\operatorname{Im} \varepsilon_{i}^{2}=\operatorname{Im} \varepsilon_{1+1} \varepsilon_{i} .
$$

So standard contractions of the two cubes $\Gamma_{i}^{\mp} \partial_{i+1}^{ \pm} a$ yield a homotopy of $\psi_{i} a \equiv_{\circ} a$ through globular maps.

It now follows that $\alpha, \beta: G_{*}^{n} \rightarrow X_{*}$ are globularly equivalent.

This proof is a higher-dimensional version of an argument in BHKP02, Sec. 6].

Corollary 2.6. The compositions in $\rho^{\square} X_{*}$ are inherited by $\rho^{\circ} X_{*}$ to give the latter the structure of globular $\omega$-groupoid.

We do not know how to prove directly that $\rho^{\circ} X_{*}$ may be given this structure of globular $\omega$-groupoid.

\section{The free globular $\omega$-groupoid on one generator}

Let $X_{*}$ be a filtered space. Then we have a diagram of maps of homotopy sets

$$
\left(\Pi X_{*}\right)_{n} \stackrel{i}{\longrightarrow}\left(\rho^{\circ} X_{*}\right)_{n} \stackrel{j}{\longrightarrow}\left(\rho^{\square} X_{*}\right)_{n} .
$$

We know from BH81b that the composition $j \circ i$ is injective. We already know that $j$ is injective. It follows that $i$ is injective. Thus the globular $\omega$-groupoid $\rho^{\circ} X_{*}$ contains the crossed complex $\Pi X_{*}$, and the results of [BH81c] show that the latter generates the former as $\omega-\mathrm{Gpd}$.

We need the following result. 
Theorem 3.1. If $G$ is a globular $\omega$-groupoid, then there is a filtered space $X_{*}$ such that $\rho^{\circ} X_{*} \cong G$.

Proof. Let $C$ be the crossed complex associated with the $\omega$-groupoid $G$ under the equivalence $(c)$ of diagram (5). By Corollary 9.3 of $[\mathbf{B H 8 1 b}$, there is a filtered space $X_{*}$ such that $\Pi X_{*} \cong C$. (Here $X$ is the classifying space $B C$ filtered by $X_{n}=B C^{(n)}$, where $C^{(n)}$ is the $n$th truncation of $C$.) It follows that $\rho^{\circ} X_{*} \cong G$.

Theorem 3.2. The globular $\omega$-groupoid $\rho^{\circ} G_{*}^{n}$ is the free globular $\omega$-groupoid on the class of the identity map, and its associated crossed complex is isomorphic to $\Pi G_{*}^{n}$.

Proof. Let $\iota: G_{*}^{n} \rightarrow G_{*}^{n}$ denote the identity map, and [ $\left.\iota\right]$ its class in $\rho^{\circ} G_{*}^{n}$. Let $H$ be a globular $\omega$-groupoid and let $x \in H_{n}$. We have to show there is a unique morphism $\alpha: \rho^{\circ} G_{*}^{n} \rightarrow H$ such that $\alpha[\iota]=x$. By Theorem 3.1 we may assume that $H$ is of the form $\rho^{\circ} X_{*}$ for some filtered space $X_{*}$; then $x$ has a representative $g: G_{*}^{n} \rightarrow X_{*}$. It follows that $\rho^{\bigcirc}(g)([\iota])=x$. This proves existence of such a morphism.

Suppose $\beta: \rho^{\circ} G_{*}^{n} \rightarrow H$ is another morphism such that $\beta([\iota])=x$. Then

$$
\gamma(\alpha), \gamma(\beta): \Pi G_{*}^{n}
$$

agree on the generating element $c^{n} \in \pi_{n}\left(G^{n}, G_{n-1}^{n}, 1\right)$ of that group. However, $\Pi G_{*}^{n}$ is generated as crossed complex by all elements $\Phi d c^{n} \in \pi_{r}\left(G_{r}^{n}, G_{r-1}^{n}, 1\right)$ for all globular face operators $d$ from dimension $n$ to dimension $r$ for $0 \leqslant r \leqslant n$. Since $\alpha, \beta$ are morphisms of $\omega$-groupoids, $\alpha\left(\Phi d c^{n}\right)=d \alpha \Phi c^{n}=d \beta \Phi c^{n}=\beta\left(d \Phi c^{n}\right)$. Therefore $\alpha, \beta$ agree on $\Pi G_{*}^{n}$, but the latter generates $\rho^{\circ} G_{*}^{n}$ as $\omega$-groupoid. So $\alpha=\beta$.

The form of this crossed complex may be deduced from the cubical Homotopy Addition Lemma [BH81a, Lemma 7.1].

$$
\delta x= \begin{cases}-x_{1}^{+}-x_{2}^{-}+x_{1}^{-}+x_{2}^{+} & \text {if } n=2, \\ -x_{3}^{+}-\left(x_{2}^{-}\right)^{u_{2} \mathbf{x}}-x_{1}^{+}+\left(x_{3}^{-}\right)^{u_{3} \mathbf{x}}+x_{2}^{+}+\left(x_{1}^{-}\right)^{u_{1} \mathbf{x}} & \text { if } n=3, \\ \sum_{i=1}^{n}(-1)^{i}\left\{x_{i}^{+}-\left(x_{i}^{-}\right)^{u_{i} \mathbf{x}}\right\} & \text { if } n \geqslant 4\end{cases}
$$

(where $u_{i}=\partial_{1}^{+} \partial_{2}^{+} \cdots \widehat{\imath} \cdots \partial_{n+1}^{+}$). In the case when $x$ is globular, this reduces to

$$
\delta x=-x_{1}^{+}+x_{1}^{-} \text {if } n \geqslant 2 .
$$

Notice that this is a groupoid formula if $n=2$.

It would be interesting to have a purely algebraic proof of this result.

\section{The higher homotopy van Kampen theorem}

Suppose for the rest of this section that $X_{*}$ is a filtered space. We suppose given a cover $\mathcal{U}=\left\{U^{\lambda}\right\}_{\lambda \in \Lambda}$ of $X_{\infty}$ such that the interiors of the sets of $\mathcal{U}$ cover $X_{\infty}$. For each $\zeta \in \Lambda^{n}$ we set $U^{\zeta}=U^{\zeta_{1}} \cap \cdots \cap U^{\zeta_{n}}, U_{i}^{\zeta}=U^{\zeta} \cap X_{i}$. Then $U_{0}^{\zeta} \subseteq U_{1}^{\zeta} \subseteq \cdots$ is called the induced filtration $U_{*}^{\zeta}$ of $U^{\zeta}$. So the globular homotopy $\omega$-groupoids in the following 
$\varrho^{\circ}$-diagram of the cover are well defined:

$$
\bigsqcup_{\zeta \in \Lambda^{2}} \varrho^{\circ} U_{*}^{\zeta} \stackrel{a}{\underset{b}{\longrightarrow}} \bigsqcup_{\lambda \in \Lambda} \varrho^{\circ} U_{*}^{\lambda} \stackrel{c}{\longrightarrow} \varrho^{\circ} X_{*} .
$$

Here $\sqcup$ denotes disjoint union (which is the same as a coproduct in the category of globular $\omega$-groupoids); $a, b$ are determined by the inclusions $a_{\zeta}: U^{\lambda} \cap U^{\mu} \rightarrow U^{\lambda}$ and $b_{\zeta}: U^{\lambda} \cap U^{\mu} \rightarrow U^{\mu}$ for each $\zeta=(\lambda, \mu) \in \Lambda^{2}$; and $c$ is determined by the inclusions $c_{\lambda}: U^{\lambda} \rightarrow X_{\infty}$.

Definition 4.1. A filtered space $X_{*}$ is said to be connected if the following conditions hold for each $n \geqslant 0$ :

- If $r>0$, the map $\pi_{0} X_{0} \rightarrow \pi_{0} X_{r}$, induced by inclusion, is surjective; i.e. $X_{0}$ meets all path connected components of all stages of the filtration $X_{r}$.

- (for $n \geqslant 1)$ : If $r>n$ and $x \in X_{0}$, then $\pi_{n}\left(X_{r}, X_{n}, x\right)=0$.

Theorem 4.2. Suppose that for every finite intersection $U^{\zeta}$ of elements of $\mathcal{U}$, the induced filtration $U_{*}^{\zeta}$ is connected. Then

(Conn) $X_{*}$ is connected;

(Iso) $c$ in the above $\varrho^{\circ}$-diagram is the coequaliser of $a, b$ in the category of globular $\omega$-groupoids.

Proof. This follows from Theorem B of [BH81b] , i.e. the analogous theorem for $\rho^{\square}$, and the fact that the equivalence from the category of globular $\omega$-groupoids to that of cubical $\omega$-groupoids with connections takes $\rho^{\circ} X_{*}$ to $\rho^{\square} X_{*}$.

Remark 4.3. If one could find convenient 'globular filtrations' of spaces, analogous to the cell decompositions of CW-complexes, then one should be able to use these results to show that $\rho^{\circ}$ of such a filtration was a free globular $\omega$-groupoid.

\section{Monoidal closed structures}

The category of cubical $\omega$-groupoids with connection is monoidal closed BH87. We recall from that paper how the tensor product is defined.

For cubical $\omega$-Gpds $F, G, H$, we define a bimorphism

$$
b: F, G \rightarrow H
$$

to be a family of functions $b=b_{p, q}: F_{p} \times G_{q} \rightarrow H_{p+q}$ such that if $x \in F_{p}, y \in G_{q}$ and $p+q=n$, then:
(i) $\partial_{i}^{\alpha} b(x, y)= \begin{cases}b\left(\partial_{i}^{\alpha} x, y\right) & \text { if } 1 \leqslant i \leqslant p \\ b\left(x, \partial_{i-p}^{\alpha} y\right) & \text { if } p+1 \leqslant i \leqslant n\end{cases}$
(ii) $\varepsilon_{i} b(x, y)= \begin{cases}b\left(\varepsilon_{i} x, y\right) & \text { if } 1 \leqslant i \leqslant p+1 \\ b\left(x, \varepsilon_{i-p} y\right) & \text { if } p+1 \leqslant i \leqslant n+1\end{cases}$ 
(iii) $\Gamma_{i} b(x, y)= \begin{cases}b\left(\Gamma_{i} x, y\right) & \text { if } 1 \leqslant i \leqslant p \\ b\left(x, \Gamma_{i-p} y\right) & \text { if } p+1 \leqslant i \leqslant n ;\end{cases}$

(iv) $b\left(x \circ_{i} x^{\prime}, y\right)=b(x, y) \circ_{i} b\left(x^{\prime}, y\right)$ if $1 \leqslant i \leqslant p$ and $x \circ_{i} x^{\prime}$ is defined in $F$;

(v) $b\left(x, y \circ_{j} y^{\prime}\right)=b(x, y) \circ_{p+j} b\left(x, y^{\prime}\right)$ if $1 \leqslant j \leqslant q$ and $y \circ_{j} y^{\prime}$ is defined in $G$.

The tensor product of cubical $\omega$-groupoids $F, G$ is given by the the universal bimorphism $F, G \rightarrow F \otimes G$ : that is any bimorphism $F, G \rightarrow H$ uniquely factors through a morphism $F \otimes G \rightarrow H$.

We next recall a result from [BH91].

Proposition 5.1. Let $X_{*}, Y_{*}$ be filtered spaces. Then there is a natural transformation

$$
\eta: \rho^{\square} X_{*} \otimes \rho^{\square} Y_{*} \rightarrow \rho^{\square}\left(X_{*} \otimes Y_{*}\right) .
$$

Proof. This natural transformation is determined by the bimorphism

$$
([f],[g]) \mapsto[f \otimes g],
$$

where $f: I_{*}^{p} \rightarrow X_{*}, g: I_{*}^{q} \rightarrow Y_{*}$. The proof that this is well defined and gives a bimorphism is routine, given the geometry of the cubes, that $I_{*}^{p} \otimes I_{*}^{q} \cong I_{*}^{p+q}$, and the well definedness of compositions on filter homotopy classes, as proved in BH81b.

It is proved in BH91, by considering the corresponding free crossed complexes, that this morphism is an isomorphism if $X_{*}, Y_{*}$ are skeletal filtrations of CW-complexes, and in BB93 that this is an isomorphism if $X_{*}, Y_{*}$ are connected and cofibred.

Because the categories of cubical and of globular $\omega$-groupoids are equivalent, and the former has a monoidal closed structure, this is inherited by the latter.

So we deduce from the above results:

Theorem 5.2. Let $X_{*}, Y_{*}$ be filtered spaces. Then there is a natural transformation

$$
\eta: \rho^{\circ} X_{*} \otimes \rho^{\circ} Y_{*} \rightarrow \rho^{\circ}\left(X_{*} \otimes Y_{*}\right)
$$

which is an isomorphism if $X_{*}, Y_{*}$ are connected and cofibred.

\section{The nerve and classifying space functors on globular $\omega$ - groupoids}

Here we just show how to define a simplicial nerve $N^{\Delta} G$ of a globular $\omega$-groupoid $G$, by the standard procedure:

$$
\left(N^{\Delta} G\right)_{n}=\omega-\operatorname{Gpd}\left(\rho^{\circ} \Delta_{*}^{n}, G\right) .
$$

The geometric realisation of this simplicial set then defines the classifying space $B G$ of $G$. However it is not so easy to see how to exploit this. The classifying space of a crossed complex is applied in, for example, BH91, BGPT, FMa07, FMP06. 


\section{Appendix A. The globular site}

We now recall from [BH81c a definition, which in Str87] and later work, is termed a globular set. This is a sequence $\left(S_{n}\right)_{n \geqslant 0}$ of sets with two families of functions

$$
\begin{aligned}
d_{i}^{ \pm}: S_{n} & \rightarrow S_{i}, i=0, \ldots, n-1, \\
s_{i}: S_{i} & \rightarrow S_{n}, i=0, \ldots, n-1,
\end{aligned}
$$

satisfying the following laws, where $\alpha, \beta= \pm$ :

(i) $d_{i}^{\alpha} d_{j}^{\beta}=d_{i}^{\alpha}$ for $i<j, \alpha, \beta= \pm$;

(ii) $s_{j} s_{i}=s_{i}$ for $i<j$;

(iii) $d_{j}^{\beta} s_{i}= \begin{cases}s_{j}^{\beta} & \text { for } j<i, \\ 1 & \text { for } j=i, \\ s_{i} & \text { for } j>i .\end{cases}$

A globular site $G S$ is a small category such that globular sets can be identified with contravariant functors $G S \rightarrow$ Set. We want to identify such a site whose objects are the globes $G^{n}$ of Section 2 , We therefore define the maps

$$
\begin{array}{rlrl}
\bar{d}_{i}^{ \pm}: G^{i} & \rightarrow G^{n}, & \bar{s}_{i}: G^{n} & \rightarrow G^{i} \\
x & \mapsto\left(0_{n-i}, \pm \sqrt{1-\|x\|^{2}}, x\right), \quad\left(x_{1}, \ldots, x_{n}\right) & \mapsto\left(x_{1}, \ldots, x_{i}\right),
\end{array}
$$

for $i<n$, where $0_{j}=\underbrace{(0, \ldots, 0)}_{j}$.

\section{Appendix B. The cubical site}

Let $K$ be a cubical set, that is, a family of sets $\left\{K_{n} ; n \geqslant 0\right\}$ with face maps

$$
\partial_{i}^{\alpha}: K_{n} \rightarrow K_{n-1}(i=1,2, \ldots, n ; \alpha=+,-)
$$

and degeneracy maps

$$
\varepsilon_{i}: K_{n-1} \rightarrow K_{n}(i=1,2, \ldots, n)
$$

satisfying the usual cubical relations:

$$
\begin{array}{rlrl}
\partial_{i}^{\alpha} \partial_{j}^{\beta} & =\partial_{j-1}^{\beta} \partial_{i}^{\alpha} & (i<j), \\
\varepsilon_{i} \varepsilon_{j} & =\varepsilon_{j+1} \varepsilon_{i} & (i \leqslant j), \\
\partial_{i}^{\alpha} \varepsilon_{j} & = \begin{cases}\varepsilon_{j-1} \partial_{i}^{\alpha} & (i<j) \\
\varepsilon_{j} \partial_{i-1}^{\alpha} & (i>j) \\
\text { id } & (i=j),\end{cases}
\end{array}
$$


We say that $K$ is a cubical set with connections if it has additional structure maps (called connections) $\Gamma_{i}^{+}, \Gamma_{i}^{-}: K_{n-1} \rightarrow K_{n}(i=1,2, \ldots, n-1)$ satisfying the relations:

$$
\begin{aligned}
& \Gamma_{i}^{\alpha} \Gamma_{j}^{\beta}=\Gamma_{j+1}^{\beta} \Gamma_{i}^{\alpha} \quad(i<j), \\
& \Gamma_{i}^{\alpha} \Gamma_{i}^{\alpha}=\Gamma_{i+1}^{\alpha} \Gamma_{i}^{\alpha}, \\
& \Gamma_{i}^{\alpha} \varepsilon_{j}= \begin{cases}\varepsilon_{j-1} \Gamma_{i}^{\alpha} & (i<j) \\
\varepsilon_{j} \Gamma_{i-1}^{\alpha} & (i>j),\end{cases} \\
& \Gamma_{j}^{\alpha} \varepsilon_{j}=\varepsilon_{j}^{2}=\varepsilon_{j+1} \varepsilon_{j} \\
& \partial_{i}^{\alpha} \Gamma_{j}^{\beta}= \begin{cases}\Gamma_{j-1}^{\beta} \partial_{i}^{\alpha} & (i<j) \\
\Gamma_{j}^{\beta} \partial_{i-1}^{\alpha} & (i>j+1),\end{cases} \\
& \partial_{j}^{\alpha} \Gamma_{j}^{\alpha}=\partial_{j+1}^{\alpha} \Gamma_{j}^{\alpha}=\mathrm{id} \\
& \partial_{j}^{\alpha} \Gamma_{j}^{-\alpha}=\partial_{j+1}^{\alpha} \Gamma_{j}^{-\alpha}=\varepsilon_{j} \partial_{j}^{\alpha} .
\end{aligned}
$$

The connections are to be thought of as extra 'degeneracies'. (A degenerate cube of type $\varepsilon_{j} x$ has a pair of opposite faces equal and all other faces degenerate. A cube of type $\Gamma_{i}^{\alpha} x$ has a pair of adjacent faces equal and all other faces of type $\Gamma_{j}^{\alpha} y$ or $\varepsilon_{j} y$.)

The prime example of a cubical set with connections is the singular cubical complex $K=S^{\square} X$ of a space $X$. Here $K_{n}$ is the set of singular $n$-cubes in $X$ (i.e. continuous maps $\left.I^{n} \rightarrow X\right)$. The face maps are induced as usual by maps $\bar{\partial}_{i}^{ \pm}: I^{n-1} \rightarrow I^{n}$ and the degeneracies by the projections $p_{i}: I^{n} \rightarrow I^{n-1}$. The connections $\Gamma_{i}^{\alpha}: K_{n-1} \rightarrow K_{n}$ are induced by the maps $\gamma_{i}^{\alpha}: I^{n} \rightarrow I^{n-1}$ defined by

$$
\gamma_{i}^{\alpha}\left(t_{1}, t_{2}, \ldots, t_{n}\right)=\left(t_{1}, t_{2}, \ldots, t_{i-1}, A\left(t_{i}, t_{i+1}\right), t_{i+2}, \ldots, t_{n}\right),
$$

where $A(s, t)=\max (s, t), \min (s, t)$ as $\alpha=-,+$, respectively.

The complex $S^{\square} X$ has some further relevant structure, namely the composition of $n$-cubes in the $n$ different directions. Accordingly, we define a cubical set with connections and compositions to be a cubical set $K$ with connections in which each $K_{n}$ has $n$ partial compositions $\circ_{j}(j=1,2, \ldots, n)$ satisfying the following axioms.

If $a, b \in K_{n}$, then $a \circ_{j} b$ is defined if and only if $\partial_{j}^{-} b=\partial_{j}^{+} a$, and then

$$
\left\{\begin{array}{l}
\partial_{j}^{-}\left(a \circ_{j} b\right)=\partial_{j}^{-} a \\
\partial_{j}^{+}\left(a \circ_{j} b\right)=\partial_{j}^{+} b,
\end{array} \quad \partial_{i}^{\alpha}\left(a \circ_{j} b\right)= \begin{cases}\partial_{j}^{\alpha} a \circ_{j-1} \partial_{i}^{\alpha} b & (i<j) \\
\partial_{i}^{\alpha} a \circ_{j} \partial_{i}^{\alpha} b & (i>j) .\end{cases}\right.
$$

The interchange laws. If $i \neq j$, then

$$
\left(a \circ_{i} b\right) \circ_{j}\left(c \circ_{i} d\right)=\left(a \circ_{j} c\right) \circ_{i}\left(b \circ_{j} d\right)
$$

whenever both sides are defined. (The diagram

$$
\left[\begin{array}{ll}
a & b \\
c & d
\end{array}\right] \underset{j}{\underset{b}{ }} \stackrel{\longrightarrow}{\longrightarrow}
$$

will be used to indicate that both sides of the above equation are defined and also to denote the unique composite of the four elements.) 
If $i \neq j$, then

$$
\begin{aligned}
& \varepsilon_{i}\left(a \circ_{j} b\right)= \begin{cases}\varepsilon_{i} a \circ_{j+1} \varepsilon_{i} b & (i \leqslant j) \\
\varepsilon_{i} a \circ_{j} \varepsilon_{i} b & (i>j),\end{cases} \\
& \Gamma_{i}^{\alpha}\left(a \circ_{j} b\right)= \begin{cases}\Gamma_{i}^{\alpha} a \circ_{j+1} \Gamma_{i}^{\alpha} b & (i<j) \\
\Gamma_{i}^{\alpha} a \circ_{j} \Gamma_{i}^{\alpha} b & (i>j),\end{cases} \\
& \Gamma_{j}^{+}\left(a \circ_{j} b\right)=\left[\begin{array}{cc}
\Gamma_{j}^{+} a & \varepsilon_{j} a \\
\varepsilon_{j+1} a & \Gamma_{j}^{+} b
\end{array}\right] \quad \underset{j+1}{\downarrow j}, \\
& \Gamma_{j}^{-}\left(a \circ_{j} b\right)=\left[\begin{array}{cc}
\Gamma_{j}^{-} a & \varepsilon_{j+1} b \\
\varepsilon_{j} b & \Gamma_{j}^{-} b
\end{array}\right] \quad \underset{j+1}{\downarrow j} .
\end{aligned}
$$

These last two equations are the transport laws ${ }^{2}$.

It is easily verified that the singular cubical complex $S^{\square} X$ of a space $X$ satisfies these axioms if $\circ_{j}$ is defined by

$$
\left(a \circ_{j} b\right)\left(t_{1}, t_{2}, \ldots, t_{n}\right)= \begin{cases}a\left(t_{1}, \ldots, t_{j-1}, 2 t_{j}, t_{j+1}, \ldots, t_{n}\right) & \left(t_{j} \leqslant \frac{1}{2}\right) \\ b\left(t_{1}, \ldots, t_{j-1}, 2 t_{j}-1, t_{j+1}, \ldots, t_{n}\right) & \left(t_{j} \geqslant \frac{1}{2}\right)\end{cases}
$$

whenever $\partial_{j}^{-} b=\partial_{j}^{+} a$.

We will now describe two graded subsets of a cubical set $K$. The globular subset $K^{\circ}$ consists in dimension $n$ of the elements $a$ such that $\partial_{i}^{\alpha} a \in \operatorname{Im} \varepsilon_{1}^{i-1}, i=1, \ldots, n$. The diskal subset $K^{D}$ consists in dimension $n$ of the elements $a$ such that $\partial_{i}^{\alpha} a \in \operatorname{Im} \varepsilon_{1}^{n-1}$ for $(\alpha, i) \neq(-, 1)$. Clearly $K^{D} \subseteq K^{\circ} \subseteq K$.

Proposition B.1. If $K$ is a cubical set with compositions, then the compositions $\circ_{i}$ are inherited by $K^{\circ}$ so that if $d_{i}^{\alpha}: K_{n}^{\circ} \rightarrow K_{n-i}^{\circ}$ is defined by $a \mapsto\left(\partial_{1}^{\alpha}\right)^{i}(a)$, then $K^{\bigcirc}$ becomes a globular set with compositions. If, further, $K$ is a cubical $\omega$-category (-groupoid), then $K^{\bigcirc}$ is a globular $\omega$-category (-groupoid).

It is proved in BH81a that if $K$ is a cubical $\omega$-groupoid, then $K^{D}$ inherits the structure of crossed complex, and in [BH81c (see also AABS02]), that $K^{\circ}$ inherits the structure of globular $\omega$-groupoid.

A globular $\omega$-category is a globular set as above with category structures $\circ_{i}$ on $S_{n}$ $0 \leqslant i \leqslant n-1$ for each $n \geqslant 0$ such that $\circ_{i}$ has $S_{i}$ as its set of objects and $D_{i}^{-}, D_{i}^{+}, E_{i}$ as its initial, final, and identity maps. These category structures must be compatible; that is:

(i) if $i>j$ and $\alpha= \pm$, then

$$
D_{i}^{\alpha}\left(x \circ_{j} y\right)=D_{i}^{\alpha} x \circ_{j} D_{i}^{\alpha} y
$$

whenever the left-hand side is defined;

\footnotetext{
${ }^{2}$ From BS76 recall that the term connection was chosen because of an analogy with pathconnections in differential geometry. In particular, the transport law is a variation or a special case of the transport law for a path-connection.
} 
(ii) $E_{i}\left(x \circ_{j} y\right)=E_{i} x \circ_{j} E_{i} y$ in $S_{n}$ whenever the left-hand side is defined;

(iii) (The interchange law) if $i \neq j$, then

$$
\left(x \circ_{j} y\right) \circ_{i}\left(z \circ_{j} w\right)=\left(x \circ_{i} z\right) \circ_{j}\left(y \circ_{j} w\right)
$$

whenever both sides are defined.

It is standard to write both sides of the interchange law (when defined) as

$$
\left[\begin{array}{cc}
x & y \\
z & w
\end{array}\right] \underset{i}{\longrightarrow j}
$$

Definition B.2. Let $K$ be a cubical set with connections and compositions. The folding operations are the operations

$$
\begin{gathered}
\psi_{i}, \Psi_{r}, \Phi_{m}: K_{n} \rightarrow K_{n} \\
\text { defined for } 1 \leqslant i \leqslant n-1,1 \leqslant r \leqslant n \text { and } 0 \leqslant m \leqslant n \text { by } \\
\psi_{i} x=\Gamma_{i}^{+} \partial_{i+1}^{-} x \circ_{i+1} x \circ_{i+1} \Gamma_{i}^{-} \partial_{i+1}^{+} x, \\
\Psi_{r}=\psi_{r-1} \psi_{r-2} \ldots \psi_{1}, \\
\Phi_{m}=\Psi_{1} \Psi_{2} \ldots \Psi_{m}=\psi_{1}\left(\psi_{2} \psi_{1}\right) \ldots\left(\psi_{m-1} \ldots \psi_{1}\right) .
\end{gathered}
$$

Note in particular that $\Psi_{1}, \Phi_{0}$ and $\Phi_{1}$ are identity operations.

Here is a picture of $\psi_{1}: K_{2} \rightarrow K_{2}$ :

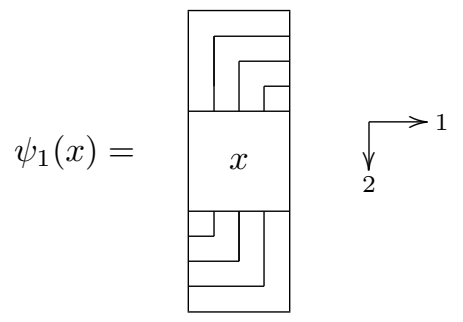

Proposition B.3. Let $K$ be a cubical set with connections and compositions. The 'folding' operator $\Phi_{n}: K_{n} \rightarrow K_{n}$ satisfies $\partial_{i}^{ \pm} \Phi_{n} x \in \operatorname{Im} \varepsilon_{1}^{i-1}$ for $1 \leqslant i \leqslant n$ and $x \in K_{n}$. That is, $\operatorname{Im} \Phi$ is contained in the globular subset of $K$.

This is part of Proposition 3.3(iii) of AABS02. Note that the compositions are needed to define $\Phi_{n}$ but this property of $\Phi_{n}$ requires only the properties (B1), (B2) giving the relations between cubical operations and connections, and does not require any axioms on the compositions.

\section{References}

[AABS02] F.A. Al-Agl, R. Brown, and R. Steiner, Multiple categories: the equivalence of a globular and a cubical approach, Adv. Math. 170 (2002), $71-118$. 
[Ash88] N. Ashley, Simplicial T-complexes and crossed complexes: a nonabelian version of a theorem of Dold and Kan, with a preface by R. Brown, Dissertationes Math. (Rozprawy Mat.) 265 (1988), 61 pp.

[BB93] H.J. Baues and R. Brown, On relative homotopy groups of the product filtration, the James construction, and a formula of Hopf, J. Pure Appl. Algebra 89 (1993), 49-61.

[Bla48] A.L. Blakers, Some relations between homology and homotopy groups, Ann. of Math. 49 (1948), 428-461.

[BBF05] V. Blanco, M. Bullejos, and E. Faro, Categorical non-abelian cohomology and the Schreier theory of groupoids, Math. Z. 251 (2005), $41-59$.

[Bro99] R. Brown, Groupoids and crossed objects in algebraic topology, Homology Homotopy Appl. 1 (1999), 1-78.

[Bro06] R. Brown, Topology and groupoids, Booksurge LLC (2006) (previous editions: McGraw Hill, 1968; Ellis Horwood, 1988.)

[BG89] R. Brown, and M. Golasiński, A model structure for the homotopy theory of crossed complexes, Cahiers Topologie Géom. Différentielle Catég. 30 (1989), 61-82.

[BGPT] R. Brown, M. Golasiński, T. Porter, and A. Tonks, Spaces of maps into classifying spaces for equivariant crossed complexes II: the general topological group case, K-Theory 23 (2001), 129-155.

[BH81a] R. Brown and P.J. Higgins, On the algebra of cubes, J. Pure Appl. Alg. 21 (1981), 233-260.

[BH81b] R. Brown and P. J. Higgins, Colimit theorems for relative homotopy groups. J. Pure Appl. Algebra 22 (1981), 11-41.

[BH81c] R. Brown and P.J. Higgins, The equivalence of $\infty$-groupoids and crossed complexes, Cahiers Topologie Géom. Différentielle 22 (1981), $371-386$.

[BH82] R. Brown and P.J. Higgins, Crossed complexes and nonabelian extensions, Category theory (Gummersbachi, 1981), Lecture Notes in Math. 962 (K. H. Kamps et al., eds.), Springer-Verlag, New York, 1982, $39-50$.

[BH87] R. Brown and P.J. Higgins, Tensor products and homotopies for $\omega$-groupoids and crossed complexes. J. Pure Appl. Algebra 47 (1987), $1-33$.

[BH90] R. Brown and P.J. Higgins, Crossed complexes and chain complexes with operators, Math. Proc. Cambridge Philos. Soc. 107 (1990), 33-57.

[BH91] R. Brown and P.J. Higgins, The classifying space of a crossed complex, Math. Proc. Cambridge Philos. Soc. 110 (1991), 95-120.

[BHS08] R. Brown, P.J. Higgins, and R. Sivera, Nonabelian algebraic topology, in preparation, http://www.bangor.ac.uk/r.brown/ nonab-a-t.html. 
[BHKP02] R. Brown, K.A. Hardie, K.H. Kamps, and T. Porter, A homotopy double groupoid of a Hausdorff space, Theory Appl. Categ. 10 (2002), 71-93.

[BL87] R. Brown and J.-L. Loday, Van Kampen theorems for diagrams of spaces, With an appendix by M. Zisman, Topology 26 (1987), 311335 .

[BP96] R. Brown and T. Porter, On the Schreier theory of non-abelian extensions: generalisations and computations, Proc. Roy. Irish Acad. Sect. A 96 (1996), 213-227.

[BS07] R. Brown and R. Sivera, Normalisation for the fundamental crossed complex of a simplicial set, J. Homotopy Related Struct., Special issue devoted to the memory of Saunders Mac Lane 2 (2007), 49-79.

[BS76] R. Brown and C.B. Spencer, Double groupoids and crossed modules, Cahiers Topologie Géom. Différentielle 17 (1976), 343-362.

[ES87] G.J. Ellis and R. Steiner, Higher-dimensional crossed modules and the homotopy groups of $(n+1)$-ads, J. Pure Appl. Algebra 46 (1987), $117-136$.

[FMa07] J. Faria Martins, On the homotopy type and fundamental crossed complex of the skeletal filtration of a CW-complex, Homology, Homotopy and Applications 9 (2007), 295-329.

[FMP06] J. Faria Martins and T. Porter, On Yetter's invariant and an extension of the Dijkgraaf-Witten invariant to categorical groups, Theory and Applications of Categories 18 (2007), 118-150.

[Jon88] D.W. Jones, A general theory of polyhedral sets and the corresponding T-complexes, Dissertationes Math. (Rozprawy Mat.) 266 (1988), $110 \mathrm{pp}$.

[Str87] R. Street, The algebra of oriented simplexes, J. Pure Appl. Algebra 49 (1987), 283-335.

Ronald Brown r.brown@bangor.ac.uk http://www.bangor.ac.uk/r.brown

School of Computer Science, University of Wales, Dean St., Bangor, Gwynedd, LL57 1UT, UK 